\title{
UBA6 and NDFIP1 regulate the degradation of ferroportin
}

Haematologica 2022

Volume 107(2):478-488

\section{Correspondence:}

DONALD B. BLOCH

dbloch@mgh.harvard.edu

LISA TRAEGER

email@lisatraeger.de

Received: February 6, 2021.

Accepted: July 22, 2021.

Pre-published: July 29, 2021

https://doi.org/10.3324/haematol.2021.278530

(C2022 Ferrata Storti Foundation

Material published in Haematologica is covered by copyright. All rights are reserved to the Ferrata Storti Foundation. Use of published material is allowed under the following terms and conditions:

https://creativecommons.org/licenses/by-nc/4.0/legalcode. Copies of published material are allowed for personal or internal use. Sharing published material for non-commercial purposes is subject to the following conditions:

https://creativecommons.org/licenses/by-nc/4.0/legalcode, sect. 3. Reproducing and sharing published material for commercial purposes is not allowed without permission in writing from the publisher.

\section{Lisa Traeger, ${ }^{1}$ Steffen B. Wiegand, ${ }^{1}$ Andrew J. Sauer, ${ }^{1}$ Benjamin H.P. Corman, ${ }^{1}$ Kathryn M. Peneyra, ${ }^{1}$ Florian Wunderer, ${ }^{1,2}$ Anna Fischbach,${ }^{1}$ Aranya Bagchi, ${ }^{1}$ Rajeev Malhotra, ${ }^{3}$ Warren M. Zapol ${ }^{1}$ and Donald B. Bloch ${ }^{1,4}$}

${ }^{1}$ Anesthesia Center for Critical Care Research of the Department of Anesthesia, Critical Care and Pain Medicine, Massachusetts General Hospital and Harvard Medical School, Boston, MA, USA; ${ }^{2}$ Department of Anesthesiology, Intensive Care Medicine and Pain Therapy, University Hospital Frankfurt, Goethe University, Frankfurt, Germany; ${ }^{3}$ Cardiovascular Research Center and the Cardiology Division of the Department of Medicine, Massachusetts General Hospital and Harvard Medical School, Boston, MA, USA and ${ }^{4}$ Division of Rheumatology, Allergy and Immunology of the Department of Medicine, Massachusetts General Hospital and Harvard Medical School, Boston, MA, USA

\section{ABSTRACT}

T Tepcidin regulates iron homeostasis by controlling the level of ferroportin, the only membrane channel that facilitates export of iron from within cells. Binding of hepcidin to ferroportin induces the ubiquitination of ferroportin at multiple lysine residues and subsequently causes the internalization and degradation of the ligand-channel complex within lysosomes. The objective of this study was to identify components of the ubiquitin system that are involved in ferroportin degradation. A HepG2 cell line, which inducibly expresses ferroportingreen fluorescent protein (FPN-GFP), was established to test the ability of small interfering (siRNA) directed against components of the ubiquitin system to prevent BMP6- and exogenous hepcidin-induced ferroportin degradation. Of the 88 siRNA directed against components of the ubiquitin pathway that were tested, siRNA-mediated depletion of the alternative E1 enzyme UBA6 as well as the adaptor protein NDFIP1 prevented BMP6- and hepcidin-induced degradation of ferroportin in vitro. A third component of the ubiquitin pathway, ARIH1, indirectly inhibited ferroportin degradation by impairing BMP6-mediated induction of hepcidin. In mice, the AAV-mediated silencing of Ndfip1 in the murine liver increased the level of hepatic ferroportin and increased circulating iron. The results suggest that the E1 enzyme UBA6 and the adaptor protein NDFIP1 are involved in iron homeostasis by regulating the degradation of ferroportin. These specific components of the ubiquitin system may be promising targets for the treatment of iron-related diseases, including iron overload and anemia of inflammation.

\section{Introduction}

Iron is an essential element that is required for a spectrum of cellular and biological processes including oxygen transport, DNA synthesis and the production of energy. High levels of iron, in the presence of oxygen, may catalyze the production of reactive oxygen species, which are free radicals that may damage cellular proteins and membranes. The level of iron in the body must be tightly regulated to provide sufficient levels to permit fundamental biological processes while preventing the damaging effects of excess iron. ${ }^{1,2}$

The hepatic hormone hepcidin is a critical regulator of systemic iron homeostasis. $^{3-5}$ Hepcidin expression is controlled by at least three stimuli: i) increased serum and liver iron, which induce hepcidin via the bone morphogenetic protein (BMP) signaling pathway; ii) increased mediators of inflammation (IL-1 $\beta$ and IL-6), which increase hepcidin via the Jak/Stat pathway; and iii) the hormone erythroferrone, which inhibits BMP signaling by sequestering BMP6 in response to increased erythropoietic demand. ${ }^{6-9}$ Hepcidin regulates iron homeostasis by controlling the cell surface level of ferroportin, which is the only known membrane channel that facilitates export of iron from within cells. ${ }^{10}$ Ferroportin is a member of the superfamily 
of transporters of small molecules, which includes more than 300 membrane-bound proteins. ${ }^{11,12}$ Ferroportin is predominantly expressed in tissues associated with iron transport including enterocytes, hepatocytes, macrophages and erythrocytes. ${ }^{10,11,13}$ The protein has 12 membrane-spanning domains, which create a channel through which iron is transported. Binding of hepcidin to the main extracellular cavity of ferroportin causes ligation of ubiquitin molecules to multiple intracellular lysine residues. ${ }^{14}$ Polyubiquitination of ferroportin induces the internalization of the hepcidinferroportin complex followed by degradation within lysosomes. ${ }^{10,14,15}$ Degradation of ferroportin results in decreased serum iron, because enterocytes, hepatocytes and macrophages are no longer able to transfer intracellular iron to the circulation. ${ }^{16}$ In addition to the ability of hepcidin to induce degradation of ferroportin, the hormone is also able to inhibit iron export by directly occluding the iron channel..$^{17}$ Occlusion of the channel may be especially important for cells, such as mature red blood cells, which lack the endocytic machinery required to degrade ferroportin. ${ }^{17}$

Ubiquitin is a 76 amino acid polypeptide that can be attached to lysine residues in proteins. The attachment of ubiquitin to a protein regulates the protein's localization, stability and/or activity. ${ }^{18}$ The process of ubiquitination involves the activation and transient conjugation of ubiquitin to a carrier protein, with subsequent final ligation of the ubiquitin molecule to a substrate. In general, ubiquitination requires three different kinds of enzymes: a ubiquitin activating enzyme (E1), a ubiquitin conjugating enzyme (E2), and a ubiquitin ligase (E3). The human ubiquitin system encodes two different E1 enzymes (UBA1 and UBA6), approximately 50 different E2 enzymes, and more than 600 E3 enzymes. ${ }^{19-21}$ The ubiquitin E3 ligases are important for substrate recognition and are divided into three different classes. ${ }^{22}$ Depending on the class of ligase, the E3 enzyme either directly transfers ubiquitin to a substrate ("HECT" and "RBR" ligases) or acts as an adaptor to facilitate the transfer of ubiquitin from an E2 enzyme directly to the substrate ("RING" E3 ligases). ${ }^{22,23}$ Binding of the E3 enzyme to the substrate may also require an adaptor protein that acts as a scaffold between the E3 enzyme and the target protein.

In this study, an in vitro small interfering RNA (siRNA) screen was performed to determine which proteins in the ubiquitin pathway are involved in ferroportin degradation. A previous study used a modified HEK293 cell line, in which expression of ferroportin was induced by the addition of ponasterone. ${ }^{24}$ Exogenous hepcidin and putative inhibitors of ferroportin degradation were added to this cell line and the level of ferroportin was then measured. To permit screening for specific enzymes involved in ferroportin ubiquitination without using exogenous hepcidin, we established a HepG2 cell line that expresses the ferroportingreen fluorescent protein (FPN-GFP) fusion protein in response to doxycycline. In this cell line, BMP6 can be used to gradually induce the expression of endogenous hepcidin. The HepG2-FPN-GFP cell line was used to show that the alternative E1 enzyme UBA6 as well as the NEDD4 family binding protein NDFIP1 are able regulate the degradation of ferroportin in response to BMP6, as well as exogenous hepcidin. Depletion of the E3 ligase ARIH1 indirectly inhibited ferroportin degradation by impairing BMP6-mediated hepcidin induction. In vivo, depletion of Ndfip1 in the murine liver increased the level of hepatic ferroportin and increased circulating iron.

\section{Methods}

\section{HepG2-FPN-GFP cell line}

A plasmid encoding human ferroportin (NM_016917) fused to GFP was a gift from Tomas Ganz (David Geffen School of Medicine, UCLA). ${ }^{14}$ DNA encoding the fusion protein was ligated into the NheI and NotI sites of plasmid pTRE2hyg (Clontech cat\#631014). The plasmid was transfected into the HepG2 "tet-on advanced" cell line (Clonetech cat\#630932) using Effectene transfection reagents (Qiagen, Germantown, MD, USA) according to the manufacturer's instructions and successfully transfected cells were selected using hygromycin $(0.4 \mathrm{mg} / \mathrm{mL})$. Individual cell lines were established and were confirmed to express FPN-GFP in the presence of doxycycline $(2 \mu \mathrm{g} / \mathrm{mL})$. One colony, which expressed a high level of FPN-GFP after treatment with doxycycline, was selected for further experiments. HepG2-FPN-GFP cells were maintained in Eagle Minimum Essential Medium, 10\% FBS, L-glutamine $(2 \mathrm{mM})$, G418 $(100 \mathrm{ng} / \mathrm{mL})$, hygromycin $(0.4 \mathrm{mg} / \mathrm{mL})$, penicillin (100 units $/ \mathrm{mL}$ ), and streptomycin $(100 \mu g)$ at $37^{\circ} \mathrm{C}$ in $5 \%$ $\mathrm{CO}_{2}$ and $95 \%$ humidity.

\section{Adeno-associated virus administration}

All experiments using mice were approved by the Partners Subcommittee on Research Animal Care at Massachusetts General Hospital, Boston, MA, USA (Protocol \# 2007N000052). Wild-type mice on a C57BL/6J background were purchased from Jackson Laboratories (Bar Harbor, ME, USA). Animals were fed a standard diet (380 ppm iron). Adeno-associated virus (AAV) short hairpin RNA (shRNA) AAV2/8-GFP-U6-m-Ndfip1-shRNA (AAV2/8-shNdfip1) and AAV2/8-GFP-U6-scrmb-shRNA (AAV2/8shControl) were obtained from Vector BioLabs (Malvern, PA, USA). Eight-week-old male mice were injected intravenously with $1 \times 10^{11}$ particles of AAV2/8-shNdfip1 or AAV2/8-shControl via the tail vein. Six weeks later, mice were anesthetized with $4 \%$ isoflurane and whole blood was collected by cardiac puncture. Liver and spleen were harvested for further analysis.

\section{Statistical analysis}

All statistical analyses were performed using GraphPad Prism 8.3.0 (GraphPad Software, San Diego, CA, USA). Data are expressed as mean \pm standard deviation (SD). The Shapiro-Wilk test was performed to test for normality. Correlation analysis were performed using Pearson correlation. Comparison of two groups was performed using the Student's $t$-test for parametric data and the Mann-Whitney-U test for non-parametric data. Comparison of more than two groups was performed using one-way ANOVA with Tukey post hoc test (for parametric data) or the Kruskal-Wallis test with Dunn's post hoc test (for non-parametric data). After adjusting for multiple comparisons, a $P$ value $<0.05$ was considered statistically significant.

A detailed description of other methods can be found in the Online Supplementary Appendix.

\section{Results}

\section{Preparation and characterization of the HepG2-FPN- GFP cell line}

Binding of hepcidin to ferroportin induces the polyubiquitination, internalization and lysosomal degradation of the ligand-channel complex. ${ }^{10}$ To identify the specific enzymes that mediate ubiquitination of ferroportin, we established a stable HepG2 cell line that inducibly expresses FPN-GFP (HepG2-FPN-GFP) in the presence of doxycycline. Treatment of HepG2-FPN-GFP cells with $2 \mu \mathrm{g} / \mathrm{mL}$ of doxy- 
cycline for 18 hours (h) induced expression of the fusion protein, which was detected at the cell surface (Figure 1A). The FPN-GFP fusion protein was able to export iron, as indirectly indicated by decreased levels of intracellular FTL and FTH1 after FPN-GFP induction (Figure 1B; Online Supplementary Figure S1A). Treatment with hepcidin (50 $\mathrm{ng} / \mathrm{mL}$ ) for 90 minutes ( $\mathrm{min}$ ) caused FPN-GFP to localize to punctate structures in the cytoplasm (Figure 1A), and treatment with hepcidin for $18 \mathrm{~h}$ caused degradation of the fusion protein (Figure 1C). Because BMP6 induces HepG2 cells to express hepcidin ${ }^{25}$ (Figure 1D), we were able to investigate the effect of gradual induction of endogenous hepcidin on ferroportin degradation. Treatment with BMP6 $(10 \mathrm{ng} / \mathrm{mL})$ for $18 \mathrm{~h}$ caused degradation of the FPN-GFP fusion protein as detected by indirect fluorescence and immunoblotting (Figure 1A and C). Pretreatment with chloroquine $(100 \mu \mathrm{M}$ for $2 \mathrm{~h})$, an inhibitor of lysosomal degradation, prevented BMP6-mediated FPN-GFP degradation and caused FPN-GFP to localize to lysosomes in the cytoplasm (Online Supplementary Figure S1B).

BMP6 induces expression of hepcidin through the BMP receptor-SMAD 1/5/8 pathway. After binding to the BMP receptor complex, activated BMP type I receptors phosphorylate SMAD 1/5/8 proteins, which translocate together with SMAD4 to the nucleus, and induce hepcidin expression. ${ }^{26}$ The siRNA-mediated inhibition of SMAD4 in HepG2-FPN-GFP cells prevented BMP6-mediated degradation of FPN-GFP (Figure 1E). Taken together, these results show that the HepG2-FPN-GFP cell line expresses inducible, functional FPN-GFP. Both BMP6-induced endogenous hepcidin and exogenous hepcidin cause internalization and degradation of the FPN-GFP fusion protein.

\section{E1 enzyme UBA6 is required for ubiquitination of ferroportin}

The human ubiquitin system encodes two E1 enzymes: UBA1 (also known as in UBE1) and UBA6. To identify the E1 enzyme that is involved in ferroportin degradation, HepG2-FPN-GFP cells were transfected with siRNA that targeted each of the E1 enzymes or with a control siRNA (siControl). Twenty-four hours after transfection with siRNA, cells were treated overnight with doxycycline to induce the expression of FPN-GFP and were then incubated with BMP6 for $18 \mathrm{~h}$. Cells that were treated with siControl and BMP6 had decreased cell surface expression of FPNGFP (Figure 2A). Depletion of UBA1 did not prevent the BMP6-induced localization of FPN-GFP to lysosomes and subsequent degradation. However, depletion of UBA6 prevented BMP6-mediated degradation of ferroportin, as indicated by the persistence of the FPN-GFP fusion protein at the cell surface (Figure 2A). Immunoblotting confirmed that depletion of UBA6, but not UBA1, impaired degradation of FPN-GFP (Figure 2B; Online Supplementary Figure 2A). The successful depletion of each of the E1 enzymes by the appropriate siRNA was confirmed by quantitative polymerase chain reaction (qPCR) (Figure 2C). Successful depletion of UBA6 by siUBA6 was not affected by the addition of BMP6 (Online Supplementary Figure S2B).

Depletion of UBA6 might block degradation of FPN-GFP or prevent BMP6-induced expression of hepcidin. To consider this latter possibility, the ability of UBA6 depletion to inhibit the BMP signal transduction pathway was investigated. Depletion of UBA6 had no effect on BMP6-mediated phosphorylation of SMAD1/5/8 (Figure 2B). In addition, siRNA directed against UBA6 did not prevent expression of endogenous hepcidin in HepG2-FPN-GFP cells (Figure 2D). In contrast, siRNA directed against SMAD4 blunted BMP6induced expression of hepcidin.

To further demonstrate that depletion of UBA6 blocks degradation of ferroportin, independent of an effect on BMP-induced expression of hepcidin, the effect of exogenous hepcidin on the cellular localization of FPN-GFP in HepG2 cells was investigated. To determine the amount of hepcidin produced by HepG2 cells after treatment with BMP6, HepG2 cells were incubated with BMP6 $(10 \mathrm{ng} / \mathrm{mL})$ for $18 \mathrm{~h}$ and the amount of hepcidin in the tissue culture medium was measured by enzyme-linked immunosorbant assay (ELISA). Under these conditions, BMP6 induced 3.9 $\mathrm{ng} / \mathrm{mL}( \pm 0.4 \mathrm{ng} / \mathrm{mL})$ of hepcidin, and this concentration of hepcidin (rather than the much higher dose of 30-50 ng/mL used in other studie ${ }^{10,24}$ ) was used to treat cells in subsequent experiments. Cells were transfected with siControl, siUBA1, or siUBA6 and treated overnight with hepcidin (4 $\mathrm{ng} / \mathrm{mL}$ ). In the presence of this low concentration of hepcidin, FPN-GFP expression at the cell surface persisted in UBA6-depleted cells but not in siControl-treated- or siUBA1-treated cells (Figure 2E). The inability of hepcidin to degrade the FPN-GFP fusion protein in siUBA6 treated cells was confirmed by immunoblot (Online Supplementary Figure S2C). Taken together, these results show that UBA6 is required for hepcidin induced internalization and degradation of FPN-GFP.

\section{The adaptor protein NDFIP1 regulates ferroportin degradation}

To identify additional components of the ubiquitin pathway that might be involved in ferroportin degradation, siRNA directed against different E2 and E3 enzymes, as well as other known components of the ubiquitin pathway, were tested for the ability to inhibit BMP6-mediated degradation of FPN-GFP (Online Supplementary Table S1, n=77). A commercially available library (Dharmacon, Lafayette, CO, USA), which contains siRNA that were previously verified to silence the corresponding targets and to minimize off-target effects, was used in these studies. HepG2 cells transfected with siRNA directed against SMAD4 were used as positive controls for inhibition of BMP6-mediated degradation of FPN-GFP; siControl was used as a negative control. Eighteen hours after treatment with BMP6, the localization of FPN-GFP was determined by immunofluorescence. In the first screen, we identified 23 siRNA directed against different E2 and E3 enzymes that appeared to block FPN-GFP relocalization to the lysosome based on FPN-GFP persistence at the cell surface after BMP6 treatment. In second and tertiary screens, all positive candidates were re-evaluated to exclude false positives.

Depletion of each of three E2 enzymes, UBE2R2, UBE2E2 and UBE2J2 partially blocked the internalization of FPNGFP (Online Supplementary Figure S3A and B), while depletion of other, individual E2 enzymes did not impair BMP6mediated FPN-GFP degradation (data not shown). Treatment with pairwise combinations of UBE2R2, UBE2E2 and UBE2J2 or all three of the E2 enzymes did not further prevent the degradation of ferroportin (data not shown), suggesting that additional E2 enzymes participate in FPN ubiquitination.

In an initial screen, depletion the NEDD family interacting protein NDFIP1 and the E3 enzyme ARIH1 impaired BMP6-induced FPN-GFP localization to lysosomes and subsequent degradation of the fusion protein (Figure $3 \mathrm{~A}$ ). 
A
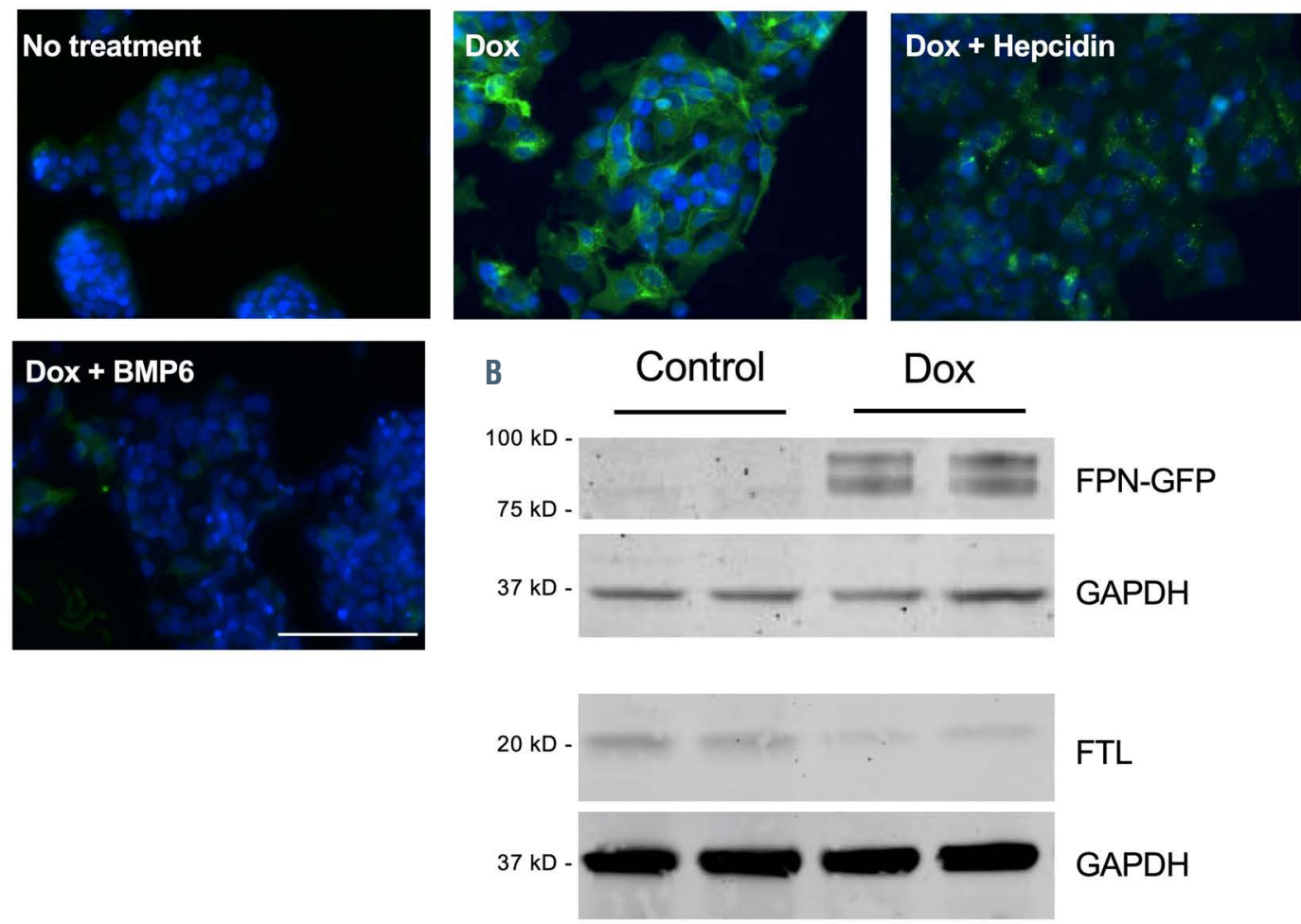

C
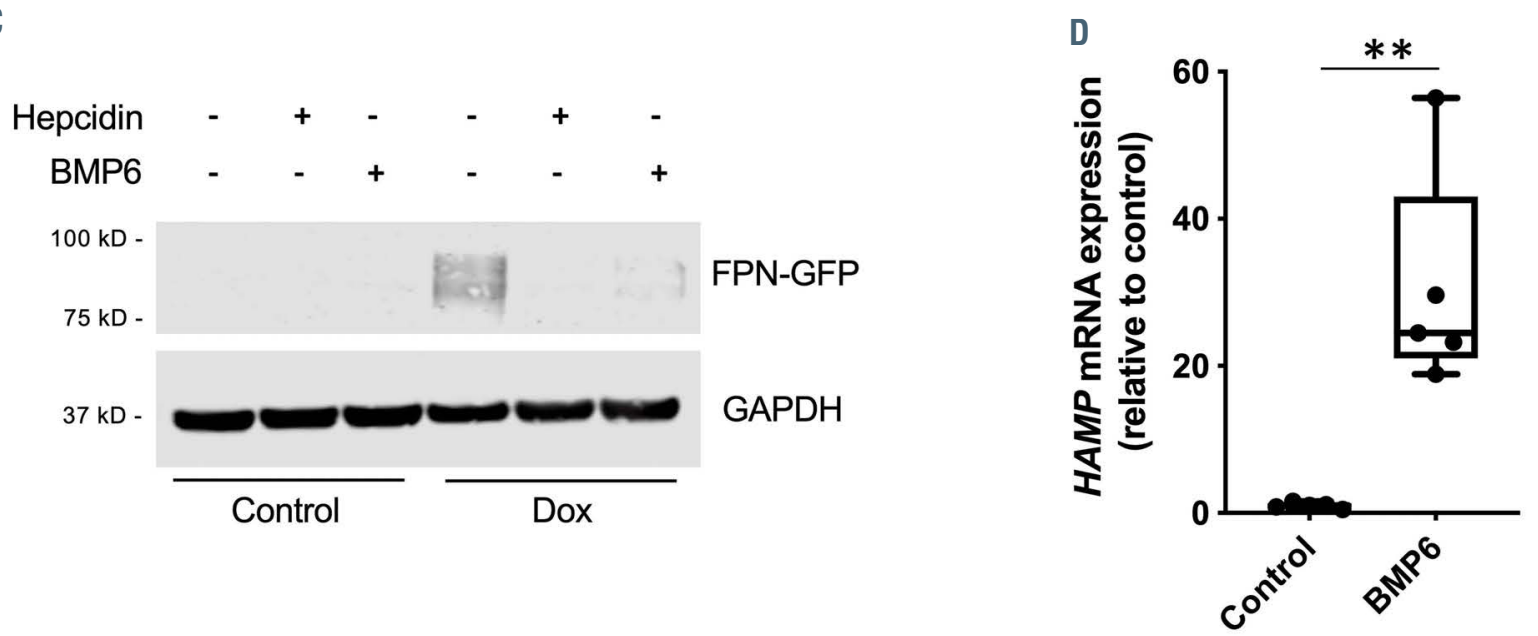

E
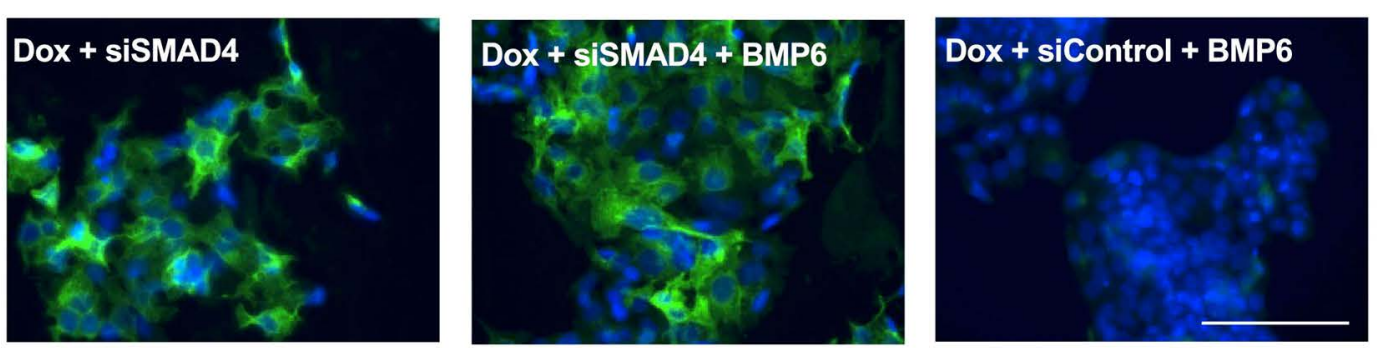

Figure 1. Characterization of the HepG2-FPN-GFP cell line. (A) Images of untreated HepG2 cells, and cells treated with doxycycline (Dox; 2 $\mu g / m L)$ alone, with Dox followed by hepcidin $(50 \mathrm{ng} / \mathrm{mL}$ ) for 90 minutes ( $\mathrm{min})$, with Dox followed by BMP6 (10 ng/mL) for 18 hours (h) are shown. (B) Treatment with Dox induced the expression of the ferroportin-green fluorescent protein (FPN-GFP) fusion protein. Dox-treated cells had reduced levels of intracellular ferritin light-chain (FTL), consistent with increased iron export in cells expressing FPN-GFP. GAPDH was used as a loading control. (C) In the absence of Dox, the fusion FPN-GFP protein was not detected by immunoblot (lanes 1 to 3). In the presence of Dox, FPN-GFP was expressed (lane 4). Treatment with hepcidin (50 ng/mL; lane 5), or BMP6 (10 ng/mL; lane 6), for $18 \mathrm{~h}$ caused degradation of the FPN-GFP fusion protein. (D) BMP6 stimulation (10 ng/mL) for $18 \mathrm{~h}$ induced hepcidin mRNA expression in HepG2-FPN-GFP cells, as determined by quantitative polymerase chain reaction (qPCR) (mRNA expression relative to control; $* * P<0.01$; Mann-Whitney-U test). (E) Images of siSMAD4 transfected cells treated with Dox (left panel), siSMAD4 transfected cells treated with Dox followed by BMP6 (10 ng/mL; middle panel) and siControl transfected cells treated with Dox followed by BMP6 (right panel) are shown. Small interfering RNA (siRNA)-mediated inhibition of SMAD4 prevented BMP6-mediated degradation of the FPN-GFP fusion protein. The location of nuclei in (A) and (E) are indicated by staining with DAPI (blue). White bar indicates $100 \mu \mathrm{m}$. 
siNDFIP1 successfully depleted NDFIP1 mRNA in both the absence (Figure $3 \mathrm{~B}$ ) and the presence (Online Supplementary Figure S3C) of exogenous BMP6. siRNA directed against NDFIP1 had no effect on the ability of BMP6 to induce hepcidin expression, demonstrating that the BMP signaling pathway was intact (Figure $3 \mathrm{C}$ ). To confirm that depletion of NDFIP1 blocks degradation of FPN-GFP, NDFIP1 deplet- ed cells were treated with exogenous hepcidin ( $4 \mathrm{ng} / \mathrm{mL})$. Compared to cells that were transfected with siControl, depletion of NDFIP1 inhibited hepcidin-mediated degradation of the FPN-GFP fusion protein (Figure 3D; Online Supplementary Figure S2C).

To investigate the possibility that NDFIP1 interacts with ferroportin, HepG2 cells were incubated in the presence or

A
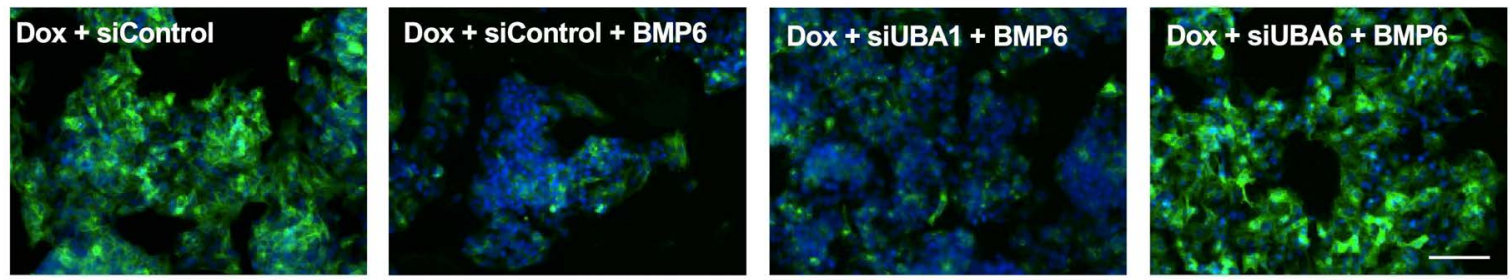

B

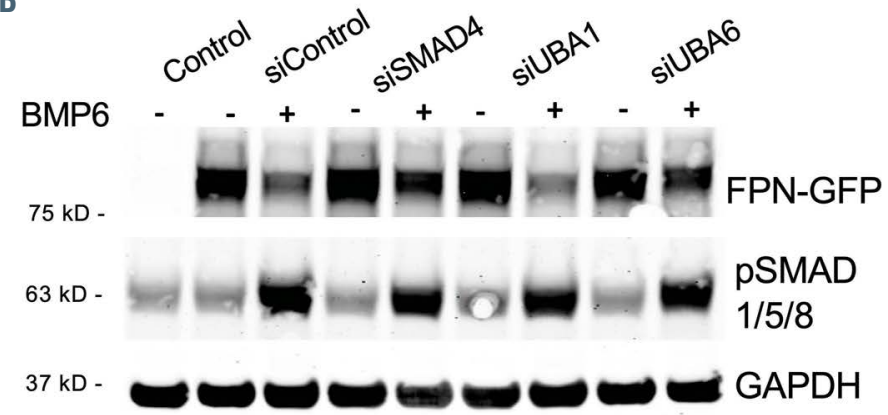

C

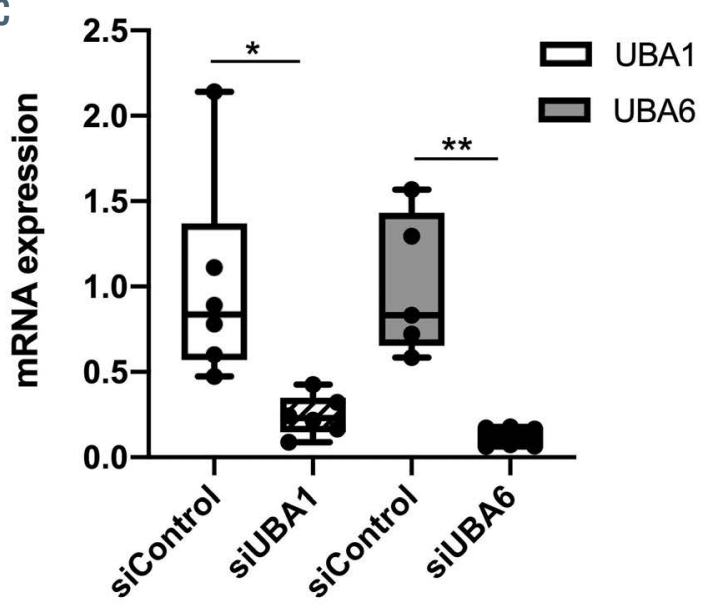

D

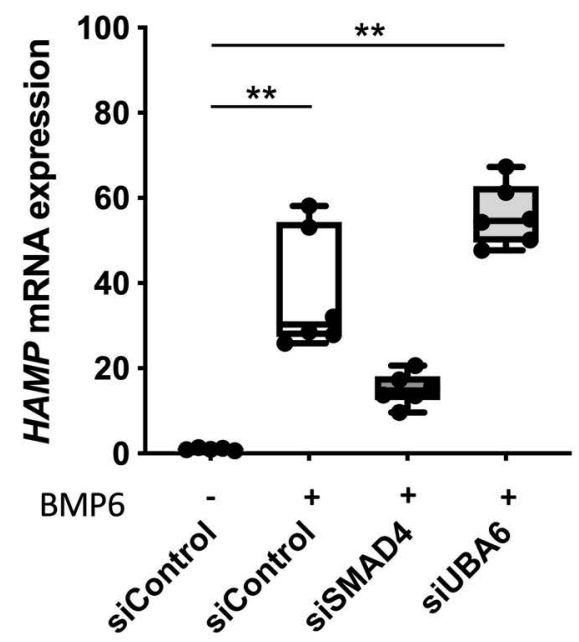

E
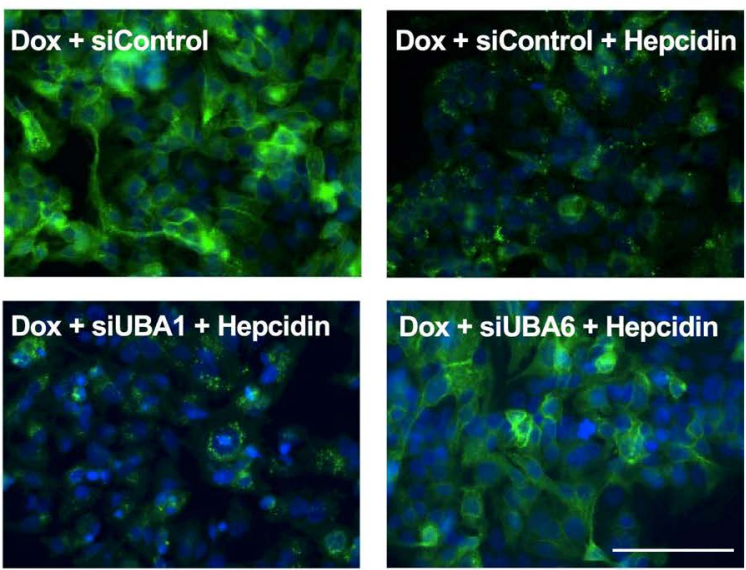

Figure 2. UBA6 is required for hepcidin-mediated degradation of the ferroportin-green fluorescent protein fusion protein. (A) Cells were transfected with siControl, siUBA1 or siUBA6, and treated with doxycycline (Dox) or Dox followed by BMP6 (10 ng/mL) for 18 hours (h), as indicated. Dox-induced expression of ferroportin-green fluorescent protein (FPN-GFP); depletion of UBA6, but not UBA1, prevented BMP6-mediated FPN degradation. (B) The levels of FPN-GFP and phosphorylated SMAD $1 / 5 / 8$ in siControl-, siUBA1- and siUBA6-transfected HepG2-FPN-GFP cells in the presence (+) or absence (-) of BMP6 (10 ng/mL for $18 \mathrm{~h})$ are shown. In the absence of Dox, control cells did not express the FPN-GFP fusion protein. Treatment with BMP6 increased the level of pSMAD 1/5/8. UBA6 depletion prevented BMP6-mediated degradation of FPN-GFP. GAPDH was used as a loading control. The immunoblot is representative of 3 separate experiments. (C) UBA1 and UBA6 were successfully depleted using the appropriate small interfering RNA (siRNA), as determined by quantitative polymerase chain reaction (qPCR) (mRNA expression relative to control; *P<0.05; $* * P<0.01$; Mann-Whitney-U test). (D) Treatment with BMP6 (10 ng/mL for $18 \mathrm{~h})$ induced the expression of hepcidin in siControl-transfected cells. Depletion of SMAD4, but not depletion of UBA6, blunted the BMP6-mediated induction of hepcidin in HepG2-FPN-GFP cells (mRNA expression relative to control; $* \star P<0.01$; Kruskal-Wallis test). (E) Images of cells transfected with siControl, siUBA1 and siUBA6 are shown. Cells were treated with Dox or Dox followed by hepcidin $(4 \mathrm{ng} / \mathrm{mL}$ for $18 \mathrm{~h})$, as indicated. Treatment with Dox induced expression of FPN-GFP in siControl treated cells, while incubation with hepcidin caused the degradation of the fusion protein. Depletion of UBA6, but not UBA1, prevented hepcidin-mediated degradation of the FPN-GFP fusion protein. The location of nuclei in (A) and (E) are indicated by staining with DAPI (blue). White bar indicates $100 \mu \mathrm{m}$. 
absence of exogenous hepcidin for $20 \mathrm{~min}$, and protein lysates were immunoprecipitated with an antibody directed against NDFIP1. In the absence of hepcidin, a small amount of FPN-GFP was detected in the immunoprecipitated protein lysate. Treatment with hepcidin caused an increase in the amount of FPN-GFP that co-immunoprecipitated with NDFIP1 (Figure 3E). Taken together, the results suggest that NDFIP1 interacts with FPN and is involved in hepcidin-induced FPN internalization and degradation.

A second adaptor protein (NDFIP2), which like NDFIP1 facilitates ubiquitination by HECT E3 enzymes, shares $79 \%$ similarity with NDFIP $1^{27}$. To investigate the potential role of NDFIP2 in the regulation of FPN, the effect of NDFIP2 depletion on BMP6-induced FPN-GFP degradation was assessed. While siNDFIP1 treatment prevented degradation of FPN-GFP, depletion of NDFIP2 had no effect on BMP6-mediated degradation of the FPN-GFP (Figure 3F; Online Supplementary Figure S3D).

NDFIP1 recruits members of the NEDD4 family of E3 ligases to target proteins. ${ }^{28}$ To investigate whether NEDD4 family members (NEDD4, NEDD4L, ITCH, WWP1, WWP2, SMURF1, SMURF2, HECW1, HECW229) regulate FPN levels, the localization of FPN-GFP in cells treated with siRNA directed against each of these enzymes was examined. None of the siRNA directed against members of the NEDD4 family, either alone or in pair-wise combinations, prevented BMP6 mediated FPN-GFP degradation (Online Supplementary Figure $S 4 A$ and $B$ ). These results indicate that either more than two of these enzymes are involved in BMP6-induced FPN degradation or additional, as yet unidentified, enzymes are able to interact with NDFIP1 and mediate FPN degradation.

\section{ARIH1 indirectly regulates ferroportin by inhibiting BMP6-mediated induction of hepcidin}

ARIH1 is a member of the Ariadne family of RBR E3 ligases. Treatment of HepG2-FPN-GFP cells with siRNA directed against ARIH1 inhibited BMP6-mediated degradation of FPN-GFP (Figure 3A). ARIH1 was successfully depleted by transfection of siARIH1 in both the absence (Figure 4A) and the presence of BMP6 (Online Supplementary Figure $54 \mathrm{C}$ ), as determined by qPCR. The addition of low dose exogenous hepcidin to HepG2-FPN-GFP cells, however, reduced the level of FPN-GFP on the surface of ARIH1depleted cells (Figure 4B). The ability of exogenous hepcidin to degrade FPN-GFP in siARIH1 treated cells was confirmed by immunoblot (Online Supplementary Figure S2C). We considered the possibility that depletion of ARIH1 inhibits FPN degradation by interfering with the ability of BMP signaling to induce hepcidin gene expression. In the absence of BMP6, the depletion of ARIH1 reduced basal hepcidin mRNA levels (Figure 4C). Depletion of siARIH1 impaired BMP6-stimulated induction of hepcidin mRNA by $80 \%$ (Figure 4D). ARIH1 depletion also inhibited BMP6-mediated induction of ID1, another target of the BMP signaling pathway (Figure 4E). Interestingly, BMP6-induced phosphorylation of SMAD1/5/8 proteins was not affected by ARIH1 depletion (Figure 4F). These results suggest that ARIH1 has an indirect effect on the stability of FPN by altering BMP6- mediated hepcidin induction through a noncanonical pathway.

The Ariadne RBR E3 ligase ARIH2 (also known as TRIAD1) is the closest relative to ARIH1 with $54 \%$ similarity. ${ }^{30}$ To consider the possibility that this second member of the Ariadne family is involved in the indirect regulation of
FPN, the effect of ARIH2 depletion on BMP6-induced FPNGFP degradation was assessed. In contrast to ARIH1, depletion of ARIH2 had no effect on BMP6-mediated degradation of the FPN-GFP protein expression (Figure 4F; Online Supplementary Figure S4D).

\section{Silencing of Ndfip1 stabilizes hepatic ferroportin in vivo}

The adaptor protein NDFIP1 was identified as a protein that is involved in FPN degradation in vitro. To address whether NDFIP1 is important for FPN degradation in vivo, mice were injected with an AAV2/8 encoding a shRNA directed against $N d f i p 1$, under the control of a U6 promoter. The AAV serotype 8 was used in these studies because it has a high efficiency of transduction in hepatocytes. ${ }^{31}$ In both AAV2/8-shNdfip1 and AAV2/8-shControl injected animals, GFP expression was detected in the liver, indicating successful systemic administration of the virus (Figure $5 \mathrm{~A}$ ). In animals injected with AAV2/8-shNdfip1, hepatic Ndfip1 mRNA levels were significantly reduced compared to control animals (Figure 5B). Mice injected with AAV2/8shNdfip1 had a 3-fold increase in FPN protein level in the liver compared to control mice (Figure 5C and D). Hamp mRNA and serum hepcidin levels were similar in both groups, suggesting that higher FPN levels were not caused by induction of hepcidin (Figure $5 \mathrm{E}$ and F). Increased hepatic FPN was associated with a $28 \%$ increase in serum iron levels in AAV2/8-shNdfip1, compared to AAV2/8-shControl, mice (Figure $5 \mathrm{G}$ ) and there was a correlation between serum iron and FPN levels (Online Supplementary Figure $S 5 A)$. Hepatic FTL levels were increased and TfR 1 mRNA was decreased in AAV2/8-shNdfip1-treated mice (Online Supplementary Figure S5B and D). As expected because of the targeting of AAV8 to the liver, ${ }^{32}$ splenic Ndfip 1 mRNA levels were not decreased in AAV2/8-shNdfip1 mice (Online Supplementary Figure S5E). The results show that the AAV2/8-mediated depletion of Ndfip1 increases the level of hepatic FPN and that Ndfip1 is required for FPN degradation in the liver.

\section{Discussion}

This study identified components of the ubiquitin system that are important for FPN degradation. A HepG2 cell line that inducibly expresses functional FPN-GFP fusion protein was established. BMP6-induced expression of hepcidin, which caused the internalization and degradation of the fusion protein and permitted analysis of FPN degradation under conditions in which the level of hepcidin increases gradually. In vitro, the alternative E1 enzyme UBA6, as well as the adaptor protein NDFIP1, were critical for hepcidininduced FPN degradation. Depletion of either UBA6 or NDFIP1 inhibited hepcidin-induced internalization and degradation of FPN-GFP. The E3 ligase ARIH1 indirectly regulated FPN stability by altering BMP6-mediated hepcidin induction through a non-canonical pathway. In vivo, the depletion of Ndfip1 in the murine liver increased the level of hepatic FPN and increased circulating iron.

In 2007, UBA6 was identified as a second ubiquitin activating E1 enzyme. UBA1 and UBA6 have non-redundant functions and each enzyme is essential for biological processes. ${ }^{33,34}$ UBA6 is widely expressed in different tissues but contributes to only approximately $1 \%$ of overall cellular ubiquitination. ${ }^{33,35}$ In addition to activating ubiquitin for 
subsequent transfer to the $\mathrm{E} 2$ enzyme, UBA6 also activates the ubiquitin-like protein FAT10, which plays a role in the immune response, obesity and aging. However, Fat10-deficient mice do not develop iron overload, ${ }^{36,37}$ suggesting that FAT10 does not play a direct role in iron homeostasis. In the present study UBA6 was found to be the E1 enzyme involved in FPN regulation in vitro; depletion of UBA6, but not UBA1, prevented hepcidin-induced FPN degradation in HepG2 cells. In contrast to UBA1, which is known to charge multiple $\mathrm{E} 2$ enzymes with ubiquitin, UBA6 transfers
A
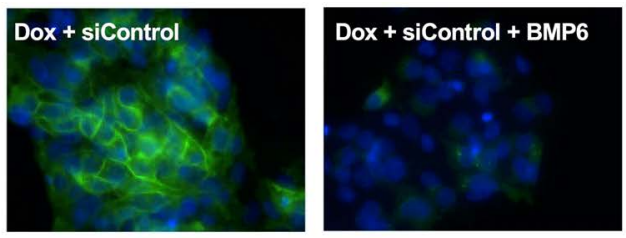

B

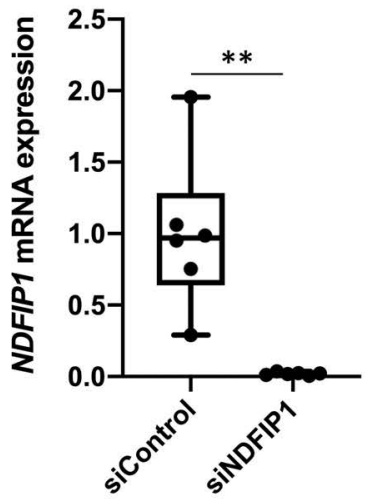

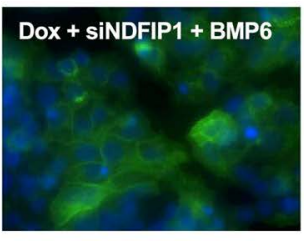

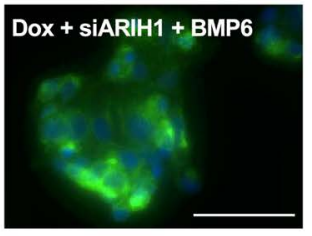

C

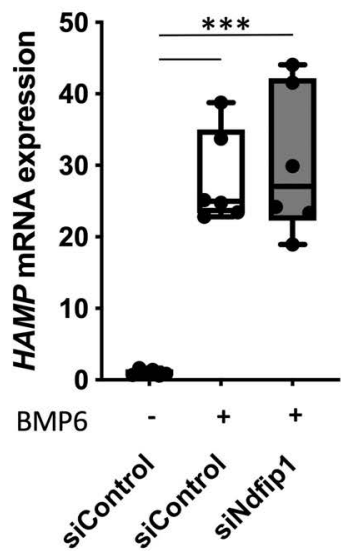

D
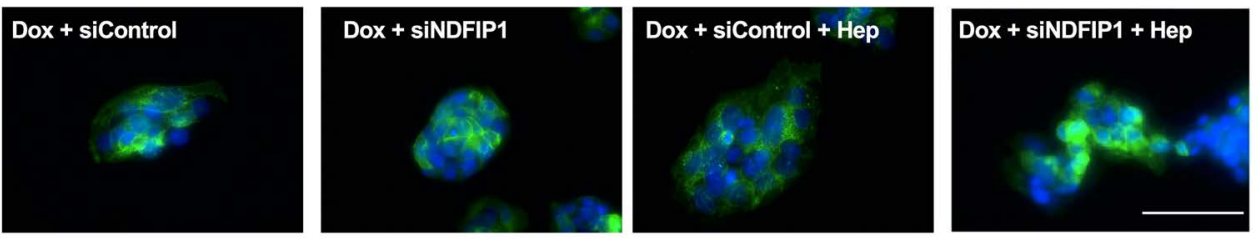

E

$\mathbf{F}$
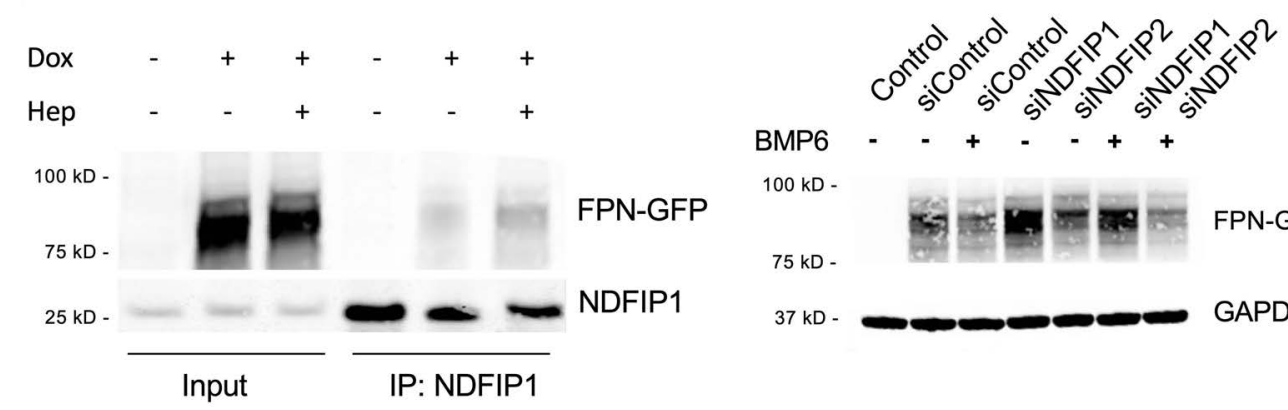

$100 \mathrm{kD}$ -

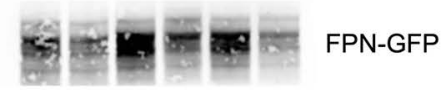

$75 \mathrm{kD}-$

GAPDH

Figure 3. NDFIP1 interacts with ferroportin and regulates ferroportin degradation. (A) Cells were transfected with siControl, siNDFIP1 or siARIH1 and treated with doxycycline (Dox) (left panel) or Dox followed by BMP6 (10 ng/mL for 18 hours [h]), as indicated. Small interfering RNA (siRNA) directed against NDFIP1 or ARIH1 prevented BMP6- mediated ferroportin-green fluorescent protein (FPN-GFP) degradation. White bar indicates $100 \mu \mathrm{m}$. (B) Transfection with siNDFIP1 successfully depleted Ndfip1, as determined by quantitative polymerase chain reaction (qPCR) (mRNA expression relative to control; $* * P<0.01$; Student's $t$-test). (C) BMP6 (10 $\mathrm{ng} / \mathrm{mL}$ for $18 \mathrm{~h}$ ) induced hepcidin expression in siControl transfected cells. Depletion of NDFIP1 did not impair the ability of BMP6 to induce the expression of hepcidin mRNA, as determined by qPCR (mRNA expression relative to control; $* * P<0.01$; One-way ANOVA and Student's $t$-test). (D) Cells were transfected with siControl or siNDFIP1 and treated with Dox or Dox followed by hepcidin $(4 \mathrm{ng} / \mathrm{mL}$ for $18 \mathrm{~h})$ as indicated. In the presence of Dox, the expression of the FPN-GFP fusion protein was induced. Treatment with hepcidin caused FPN-GFP internalization and its subsequent degradation in siControl-treated cells, but not in siNDFIP1-treated cells. White bar indicates $100 \mu \mathrm{m}$. (E) A low level of FPN-GFP co-immunoprecipitated with NDFIP1 in Dox-treated HepG2-FPN-GFP cells that were not treated with hepcidin The level of FPN co-immunoprecipitating with NDFIP1 increased after treatment with hepcidin ( $50 \mathrm{ng} / \mathrm{mL}$ ) for 20 minutes (min). Immunoprecipitation was performed using rabbit anti-NDFIP1 antibody. FPN-GFP was detected using a mouse anti-GFP antibody. The immunoblot is representative of 3 separate experiments. (F) HepG2FPN-GFP cells were transfected with siControl-, siNDFIP1- or siNDFIP2 and incubated in the presence (+) or absence (-) of BMP6 (10 ng/mL for 18h). In the absence of Dox, control cells did not express the FPN-GFP fusion protein. (siRNA) directed against NDFIP1, but not NDFIP2, prevented BMP6-induced degradation of the FPNGFP fusion protein, as determined by immunoblot. GAPDH was used as a loading control. The immunoblot is representative of 4 separate experiments. 
A

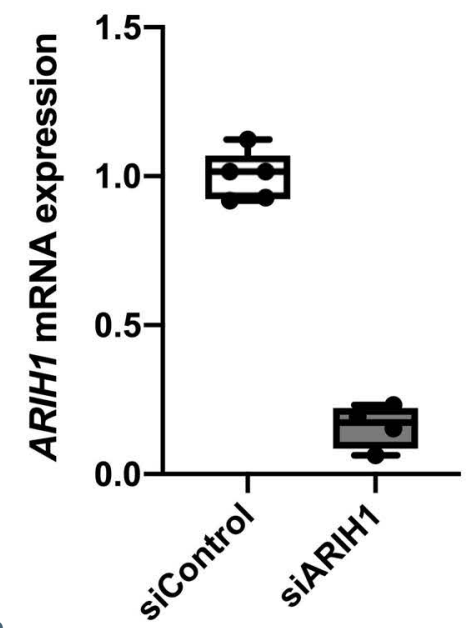

C

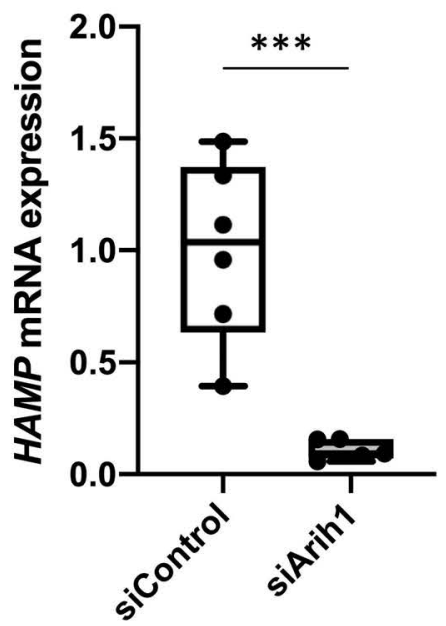

B
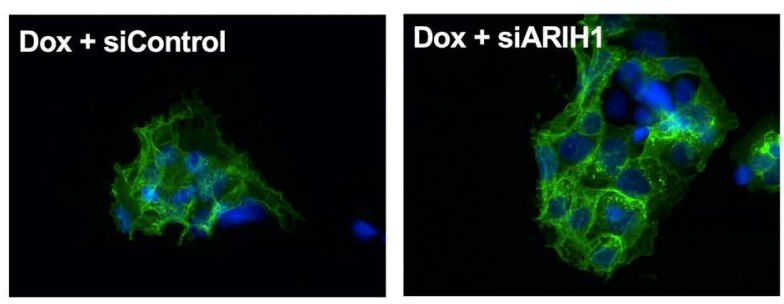

Dox + siControl + Hep
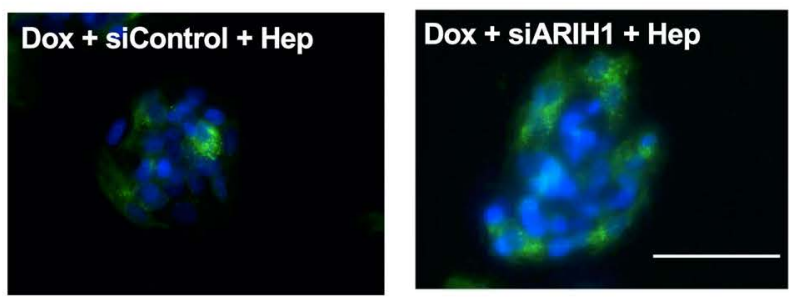

D

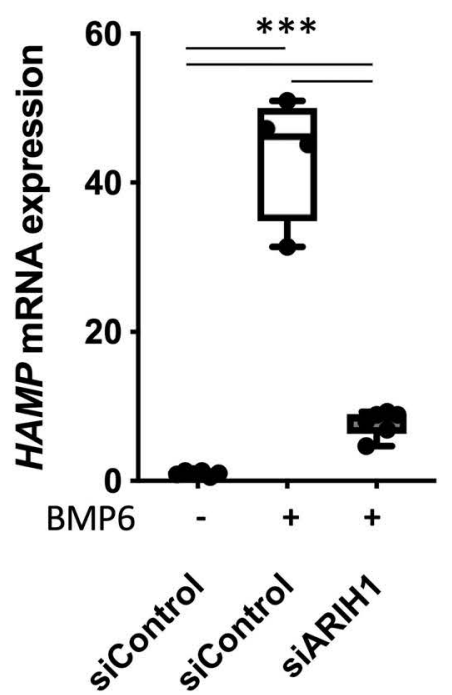

E
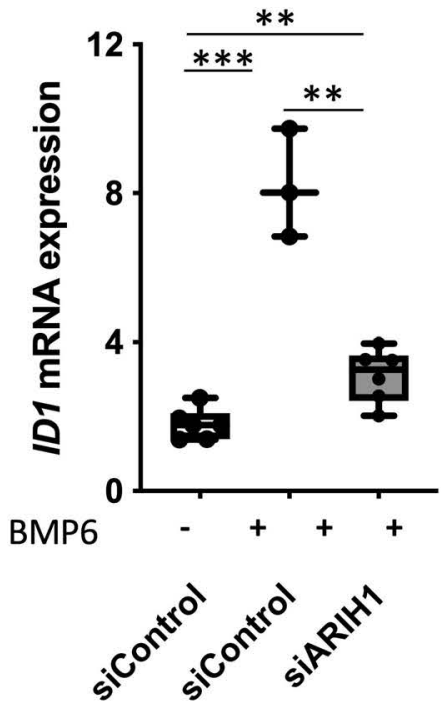

$\mathbf{F}$

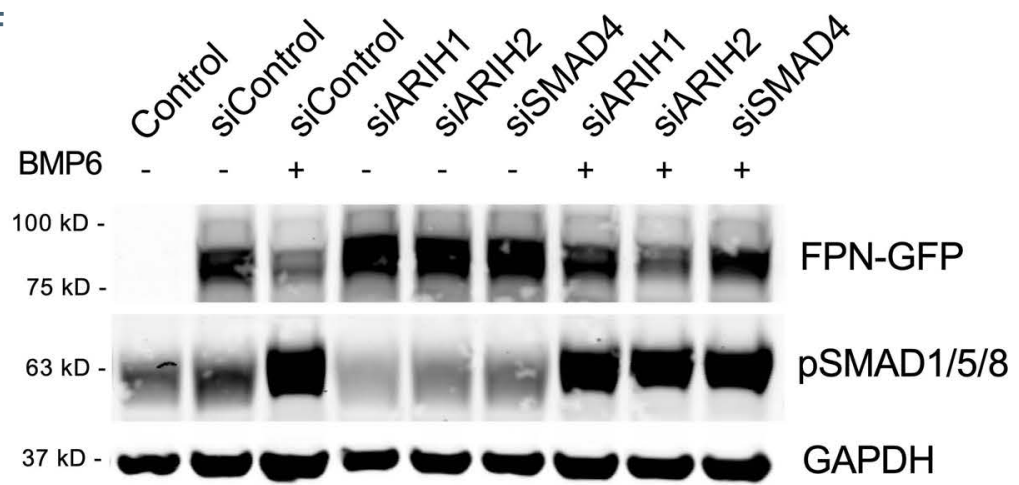

Figure 4. ARIH1 regulates BMP6-mediated induction of hepcidin. (A) Transfection with siARIH1 successfully depleted ARIH1 in HepG2-FPN-GFP cells, as determined by quantitative polymerase chain reaction (qPCR) (mRNA expression relative to control; $* * * P<0.001$; Student's $t$-test). (B) HepG2-FPN-GFP cells were transfected with siControl or siARIH1 and were treated with doxycycline (Dox) or Dox followed by hepcidin ( $4 \mathrm{ng} / \mathrm{mL}$ for 18 hours [h]) as indicated. In the presence of Dox, the expression of the ferroportin-green fluorescent protein (FPN-GFP) fusion protein was induced. Treatment with hepcidin caused FPN-GFP localization to lysosomes and its subsequent degradation in siControl-treated cells as well as in siARIH1-treated cells. White bar indicates $100 \mu \mathrm{m}$. (C) Treatment of HepG2 cells with siARIH1 reduced the basal expression of hepcidin mRNA, as determined by qPCR (mRNA expression relative to control; $* * * P \leq 0.001$; Student's $t$-test). (D) Pretreatment of HepG2 cells with small interfering RNA (siRNA) directed against ARIH1 reduced BMP6-mediated hepcidin mRNA expression (relative to control), as determined by qPCR $(* * *=P \leq 0.001$; One-way ANOVA). (E) BMP6 $(10 \mathrm{ng} / \mathrm{mL}$ for $18 \mathrm{~h})$ induced the expression of ID1 in siControl transfected cells. Pretreatment of HepG2 cells with siRNA directed against ARIH1 blunted BMP6 induced expression of ID1, as determined by qPCR (mRNA expression relative to control; ** $P<0.01$; $* * * P<0.001$; Oneway ANOVA). (F) Immunoblot showing levels of FPN-GFP and phosphorylated SMAD1/5/8 in siARIH1- or siARIH2-transfected cells in the presence (+) or absence $(-)$ of BMP6 (10 ng/mL for $18 \mathrm{~h}$ ). The level of pSMAD 1/5/8 was increased in all BMP6-treated cells. siRNA directed against ARIH1, but not ARIH2, prevented BMP6induced degradation of the FPN-GFP fusion protein. GAPDH was used as a loading control. The immunoblot is representative of 4 separate experiments. 
A

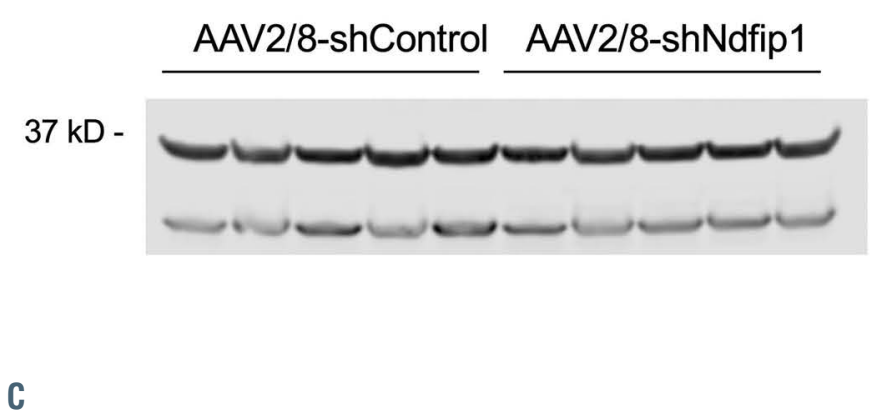

B

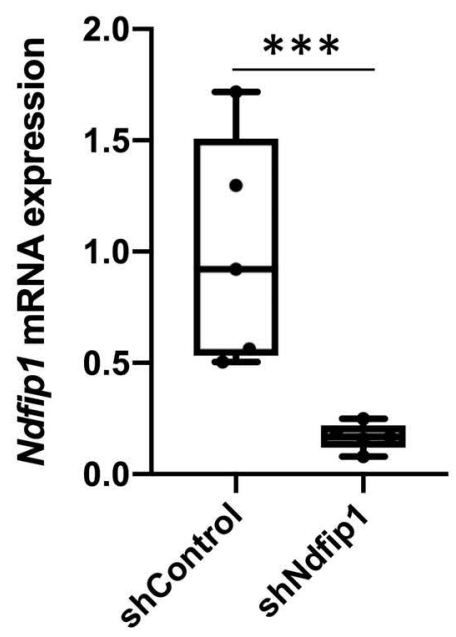

D

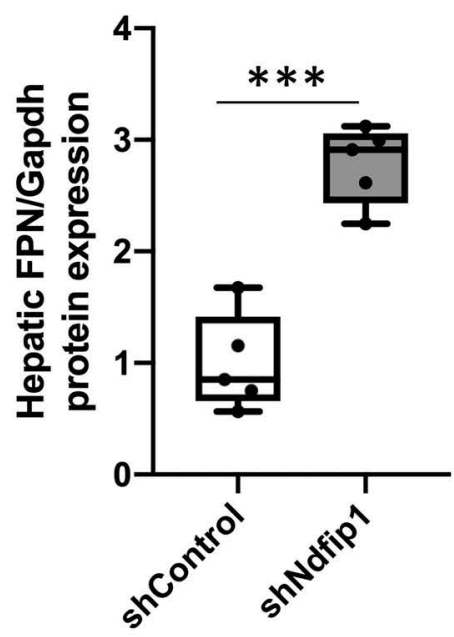

F

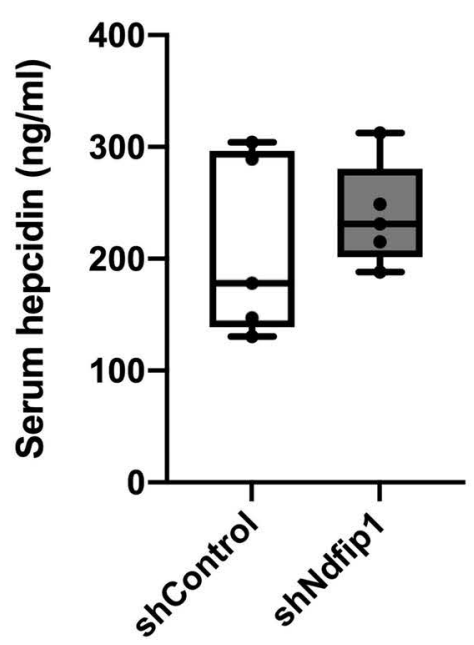

E

GAPDH

GFP

\section{GAPDH}

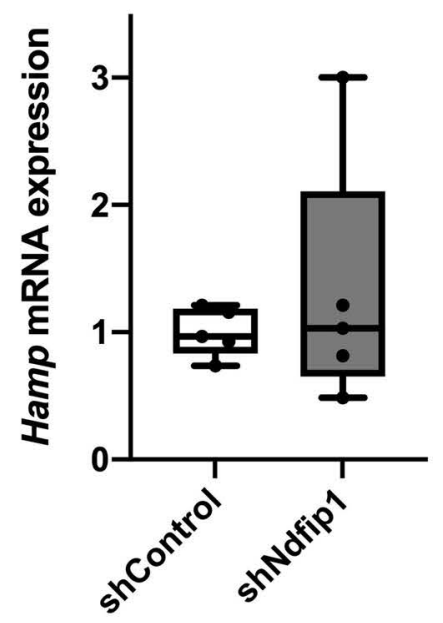

G

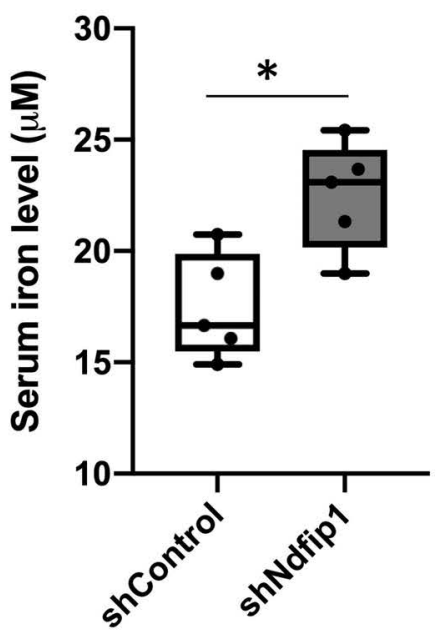

Figure 5. Ndfip1 regulates ferroportin in vivo. (A) Green fluorescent protein (GFP) was detected in the liver of AAV2/8-shControl- and AAV2/8-shNdfip1-injected mice. GAPDH was used as a loading control. (B) AAV2/8-shNdfip1 reduced the level of Ndfip1 mRNA in the liver, as determined by quantitative polymerase chain reaction (qPCR) (mRNA expression relative to control; **P<0.01; Student's $t$-test). (C) The level of ferroportin (FPN) in the liver of AAV2/8-shNdfip1-treated mice was increased compared to AAV2/8-shControl-treated mice, as determined by immunoblot. GAPDH was used as a loading control. (D) Densitometric analysis of immunoblot in (C) ( $* * * P<0.001$; Student's $t$-test). (E) HAMP mRNA expression was similar in the liver of AAV2/8-shNdfip1-treated mice compared to AAV2/8-shControl-treated mice as determined by qPCR (mRNA expression relative to control). (F) Serum hepcidin levels were similar in AAV2/8-shNdfip1- and AAV2/8-shControl-treated mice as determined by enzyme-linked immunosorbant assay. (G) Serum iron levels were increased in mice treated with AAV2/8-shNdfip1 (*P<0.05; Student's $t$-test). 
ubiquitin to a small number of E2 enzymes. ${ }^{35}$ Although some E2 enzymes interact with both UBA1 and UBA6, one E2 enzyme (USE1, also known as UBE2Z) is exclusively charged by $\mathrm{UBA} 6^{35,38}$. In this study, we found that inhibition of USE1 did not interfere with hepcidin-induced FPN degradation (data not shown), indicating that an E2 enzyme other than (or in addition to) USE1 is involved in FPN regulation. In UBA6-depleted cells, UBA1 failed to induce hepcidin-mediated FPN degradation, indicating non-redundant functions of UBA1 and UBA6 in FPN regulation. The results suggest that an as yet unidentified E2 enzyme, exclusively charged by UBA6, plays a role in FPN degradation.

Members of the NEDD4 family of HECT-type E3 ligases contain a "WW" domain that interacts with a proline rich PPXY (PY) motif in the target protein. However, some target proteins lack a PY domain and ubiquitination of these proteins requires the presence of adaptor proteins NDFIP1 or NDFIP2 to act as a scaffold between the two proteins. NDFIP proteins contain three transmembrane domains as well as two PY motifs, which interact with the WW domain of several members of the NEDD4 family of E3 ligases. ${ }^{28}$ In this study, NDFIP1 was shown to interact with FPN in HepG2 cells in vitro and regulates the level of FPN in the liver in vivo. None of the WW domain-containing NEDD4 family members that were tested individually or in pair-wise combination prevented BMP6-induced FPN degradation. The results suggest that several NEDD4 family members may have a redundant role in FPN degradation. Another possibility is that an as yet unknown E3 ligase interacts with the adaptor protein NDFIP1 to ubiquitinate FPN.

ARIH1 is a member of the Ariadne family of E3 RBR ligase. ARIH1 is highly expressed in the nucleus, where it interacts with Cajal and PML nuclear bodies. ${ }^{39}$ ARIH1 associates with neddylated Cullin-RING E3 ligases (CRL) and monoubiquitinates CRL targets. ${ }^{40}$ In this study, ARIH1 was shown to indirectly regulate FPN stability by altering BMP6-mediated hepcidin induction through a non-canonical pathway. Depletion of ARIH1 blunted basal, as well as BMP6-mediated, hepcidin and ID1 mRNA expression without altering the phosphorylation of SMAD $1 / 5 / 8$ proteins in response to BMP6. Further studies are needed to elucidate the mechanism as to how ARIH1 regulates hepcidin expression in response to BMP6.

NDFIP1 was previously shown to have a role in iron homeostasis. ${ }^{41-43}$ NDFIP1 binds to divalent metal transporter 1 (DMT1), the major iron transporter for non-heme iron import. ${ }^{44}$ NDFIP1 recruits the NEDD4 family member WWP2 to ubiquitinate DMT1 ${ }^{43}$. In vivo, Ndfip1 is involved in the regulation of DMT1 in enterocytes. ${ }^{41}$ The expression of Dmt1 in enterocytes of Ndfip 1 deficient mice is increased under normal iron conditions as well as during iron deficiency. The increased level of Dmt1 leads to increased iron absorption, and under normal dietary iron conditions Ndfip1-deficient mice develop a phenotype resembling classic hereditary hemochromatosis, with increased hepatic, duodenal and serum iron levels. ${ }^{43,45}$ In this study we show that depletion of Ndfip1 in the liver increased the level of FPN. Ndfip1 appears to regulate two steps in iron metabolism: iron import by DMT1 in enterocytes and iron export by FPN in the liver. Ndfip1-deficient mice were not used in this study, because Ndfip 1 deficiency results in a severe inflammatory phenotype caused by hyperactivation of $\mathrm{T}$ cells. ${ }^{45,46}$

In summary, this study demonstrated that the E1 enzyme UBA6 and the adaptor protein NDFIP1 are important for iron homeostasis, regulating the degradation of hepatic FPN. In the future, it may be possible to target specific components of the ubiquitin pathway with small molecules; ${ }^{47}$ the results of this study may offer novel approaches to treating disorders of iron metabolism.

\section{Disclosures}

No conflicts of interest to disclose.

\section{Contributions}

$L T, F W$ and $D B B$ designed and conceived the study; $L T$ and $D B B$ wrote and edited the manuscript; $L T, S B W, A J S, B H P C, K P$, $A F$ and $D B B$ performed experiments; $L T, R M, A B$ and $D B B$ analyzed and interpreted the data; $D B B$ and WMZ supervised the study. All authors approved the final version for submission.

\section{Funding}

This study was supported by Luisa Hunnewell and Larry Newman (DBB), the German Research Foundation (TR 1642/11 to $L T$, WI 5162/2-1 to SBW, Wu 841/1-1 to FW, FI 2429/1-1 to AF), NIH (R01HL142809 to RM, R01DK125786 to AB) and the American Heart Association (18TPA34230025 to RM) $20 I O A 35360009$ to $A B)$.

\section{References}

1. Salahudeen AA, Bruick RK. Maintaining mammalian iron and oxygen homeostasis: sensors, regulation, and cross-talk. Ann N Y Acad Sci. 2009;1177:30-38.

2. Pantopoulos K, Porwal SK, Tartakoff A, Devireddy L. Mechanisms of mammalian iron homeostasis. Biochemistry. 2012;51(29):5705-5724.

3. Pigeon C, Ilyin G, Courselaud B, et al. A new mouse liver-specific gene, encoding a protein homologous to human antimicrobial peptide hepcidin, is overexpressed during iron overload. J Biol Chem. 2001;276(11):7811-7819.

4. Nicolas G, Viatte L, Lou D-O, et al. Constitutive hepcidin expression prevents iron overload in a mouse model of hemochromatosis. Nat Genet. 2003;34(1):97101.

5. Park CH, Valore EV, Waring AJ, Ganz T. Hepcidin, a urinary antimicrobial peptide synthesized in the liver. I Biol Chem. 2001;276(11):7806-7810

6. Dev S, Babitt JL. Overview of iron metabolism in health and disease. Hemodial Int. 2017;21(Suppl 1):S6-S20.

7. Sebastiani G, Wilkinson N, Pantopoulos K. Pharmacological targeting of the hepcidin/ferroportin axis. Front Pharmacol. 2016;7:160.

8. Wang C-Y, Xu Y, Traeger L, et al. Erythroferrone lowers hepcidin by sequestering $\mathrm{BMP} 2 / 6$ heterodimer from binding to the BMP type I receptor ALK3. Blood. 2020;135(6):453-456.

9. Wunderer F, Traeger L, Sigurslid $\mathrm{HH}$ Meybohm P, Bloch DB, Malhotra R. The role of hepcidin and iron homeostasis in atherosclerosis. Pharmacol Res. 2020;153:104664.

10. Nemeth E, Tuttle MS, Powelson I, et al. Hepcidin regulates cellular iron efflux by binding to ferroportin and inducing its internalization. Science. 2004;306(5704):2090
2093

11. Drakesmith H, Nemeth E, Ganz T. Ironing out ferroportin. Cell Metab. 2015;22(5):777787

12. Lin L, Yee SW, Kim RB, Giacomini KM. SLC transporters as therapeutic targets: emerging opportunities. Nat Rev Drug Discov. 2015;14(8):543-560

13. Zhang D-L, Ghosh MC, Ollivierre H, Li Y, Rouault TA. Ferroportin deficiency in erythroid cells causes serum iron deficiency and promotes hemolysis due to oxidative stress. Blood. 2018;132(19):2078-2087.

14. Qiao B, Sugianto P, Fung E, et al. Hepcidininduced endocytosis of ferroportin is dependent on ferroportin ubiquitination. Cell Metab. 2012;15(6):918-924.

15. Ross SL, Tran L, Winters A, et al. Molecular mechanism of hepcidin-mediated ferroportin internalization requires ferroportin lysines, not tyrosines or JAK-STAT. Cell Metab. 2012;15(6):905-917. 
16. Camaschella C, Nai A, Silvestri L. Iron metabolism and iron disorders revisited in the hepcidin era. Haematologica. 2020;105 (2):260-272.

17. Aschemeyer S, Qiao B, Stefanova D, et al. Structure-function analysis of ferroportin defines the binding site and an alternative mechanism of action of hepcidin. Blood. 2018;131(8):899-910.

18. Pickart CM, Eddins MJ. Ubiquitin: structures, functions, mechanisms. Biochim Biophys Acta. 2004;1695(1-3):55-72

19. Morreale FE, Walden $\mathrm{H}$. Types of ubiquitin ligases. Cell. 2016;165(1):248-248.

20. hen M, Schmitt S, Buac D, Dou OP. Targeting the ubiquitin-proteasome system for cancer therapy. Expert Opin Ther Targets. 2013;17(9):1091-1108.

21. Stewart MD, Ritterhoff T, Klevit RE, Brzovic PS. E2 enzymes: more than just middle men. Cell Res. 2016;26(4):423-440.

22. Ardley HC, Robinson PA. E3 ubiquitin ligases. Essays Biochem. 2005;41:15-30.

23. Weber J, Polo S, Maspero E. HECT E3 ligases: a tale with multiple facets. Front Physiol. 2019;10:370.

24. Fung E, Sugianto P, Hsu J, Damoiseaux R, Ganz T, Nemeth E. High-throughput screening of small molecules identifies hepcidin antagonists. Mol Pharmacol. 2013;83(3):681690.

25. Meynard D, Kautz L, Darnaud V, CanonneHergaux F, Coppin H, Roth M-P. Lack of the bone morphogenetic protein BMP6 induces massive iron overload. Nat Genet. 2009;41(4):478-481.

26. Xiao X, Alfaro-Magallanes VM, Babitt JL. Bone morphogenic proteins in iron homeostasis. Bone. 2020;138:115495

27. Shearwin-Whyatt LM, Brown DL, Wylie FG, Stow JL, Kumar S. N4WBP5A (Ndfip2), a Nedd4-interacting protein, localizes to multivesicular bodies and the Golgi, and has a potential role in protein trafficking. J Cell Sci. 2004;117(Pt 16):3679-3689.

28. Harvey KF, Shearwin-Whyatt LM, Fotia A Parton RG, Kumar S. N4WBP5, a potential target for ubiquitination by the Nedd4 family of proteins, is a novel Golgi-associated protein. J Biol Chem. 2002;277(11):93079317.

29. Ingham RJ, Gish G, Pawson T. The Nedd 4 family of E3 ubiquitin ligases: functional diversity within a common modular architecture. Oncogene. 2004;23(11):1972-1984.

30. Kelsall IR, Duda DM, Olszewski JL, et al. TRIAD 1 and HHARI bind to and are activated by distinct neddylated Cullin-RING ligase complexes. EMBO J. 2013;32(21):2848-2860.

31. Nam H-J, Lane MD, Padron E, et al. Structure of adeno-associated virus serotype 8 , a gene therapy vector. J Virol. 2007;81(22):1226012271.

32. Snyder RO, Miao CH, Patijn GA, et al Persistent and therapeutic concentrations of human factor IX in mice after hepatic gene transfer of recombinant $\mathrm{AAV}$ vectors. Nat Genet 1997;16(3):270-276.

33. Barghout SH, Schimmer AD. E1 Enzymes as Therapeutic Targets in Cancer. Pharmacol Rev. 2021;73(1):1-56.

34. Groettrup M, Pelzer C, Schmidtke G, Hofmann K. Activating the ubiquitin family: UBA6 challenges the field. Trends Biochem Sci. 2008;33(5):230-237.

35. Jin J, Li X, Gygi SP, Harper JW. Dual E1 activation systems for ubiquitin differentially regulate E2 enzyme charging. Nature. 2007;447(7148):1135-1138.

36. Canaan A, Yu X, Booth CJ, et al. FAT10/diubiquitin-like protein-deficient mice exhibit minimal phenotypic differences. Mol Cell Biol. 2006;26(13):5180-5189.

37. Canaan A, DeFuria J, Perelman E, et al. Extended lifespan and reduced adiposity in mice lacking the FAT10 gene. Proc Natl Acad
Sci U S A. 2014;111(14):5313-5318.

38. Wang F, Zhao B. UBA6 and its bispecific pathways for ubiquitin and FAT10. Int J Mol Sci. 2019;20(9):2250.

39. Elmehdawi F, Wheway G, Szymanska K, et al. Human homolog of drosophila Ariadne (HHARI) is a marker of cellular proliferation associated with nuclear bodies. Exp Cell Res. 2013;319(3):161-172.

40. Scott DC, Rhee DY, Duda DM, et al. Two distinct types of E3 ligases work in unison to regulate substrate ubiquitylation. Cell. 2016:166(5):1198-1214.

41. Foot NJ, Leong YA, Dorstyn LE, et al. Ndfip1deficient mice have impaired DMT1 regulation and iron homeostasis. Blood. 2011;117(2):638-646.

42. Foot NJ, Gembus KM, Mackenzie K, Kumar S. Ndfip2 is a potential regulator of the iron transporter DMT1 in the liver. Sci Rep. 2016;6:24045

43. Foot NJ, Dalton HE, Shearwin-Whyatt LM et al. Regulation of the divalent metal ion transporter DMT1 and iron homeostasis by a ubiquitin-dependent mechanism involving Ndfips and WWP2. Blood. 2008;112(10): 4268-4275.

44. Yanatori I, Kishi F. DMT1 and iron transport. Free Radic Biol Med. 2019;133:55-63.

45. Oliver PM, Cao X, Worthen GS, et al. Ndfip1 protein promotes the function of itch ubiquitin ligase to prevent $\mathrm{T}$ cell activation and $\mathrm{T}$ helper 2 cell-mediated inflammation. Immunity. 2006;25(6):929-940.

46. Nemeth E, Valore EV, Territo M, Schiller G, Lichtenstein A, Ganz T. Hepcidin, a putative mediator of anemia of inflammation, is a type II acute-phase protein. Blood. 2003;101(7):2461-2463.

47. Deng L, Meng T, Chen L, Wei W, Wang P. The role of ubiquitination in tumorigenesis and targeted drug discovery. Signal Transduct Target Ther. 2020;5(1):11 